\title{
Reconstruction and Subcellular Localization Analysis of Eriocheir sinensis Molting Protein- Protein Interaction Network
}

\author{
Bin Wang', ${ }^{1,2}$ Qianji Ning ${ }^{1, *}$, Qian Wang ${ }^{2}$, Wei Peng' ${ }^{2}$, Tong Hao ${ }^{2, *}$ and Jinsheng Sun ${ }^{2, *}$ \\ ${ }^{1}$ College of Life Sciences, Henan Normal University, Xinxiang 453007, Henan, China \\ ${ }^{2}$ Tianjin Key Laboratory of Animal and Plant Resistance/College of Life Sciences, \\ Tianjin Normal University, Tianjin 300387, China
}

\begin{abstract}
A B S T R A C T
Molting abnormalities of Chinese Mitten Crab (Eriocheir sinensis) can seriously affect their growth, causing significant losses to the aquaculture industry. The current studies on the molting mechanism mainly focused on the independent analysis of single protein, rather than a protein-protein interaction. In this work, with the systematic point of view, the subcellular location of 830 unidentified proteins were annotated based on the previously reconstructed protein-protein interaction network (PIN) of $E$. sinensis using the bioinformatics analysis method, which accounts for $91.9 \%$ of all un-localized proteins in the network. Subsequently, the molting interaction network was extracted from the global PPI, which contains 35 proteins and 32 interactions. By analyzing the subcellular annotation of the molting interaction network, the subcellular localization of 12 proteins was obtained. Half of these proteins located on the cell membrane, indicating the close relationship between molting process and the changes of membrane. The results of this work provide a theoretical basis of the further elucidation of the molting mechanism for E. sinensis.
\end{abstract}
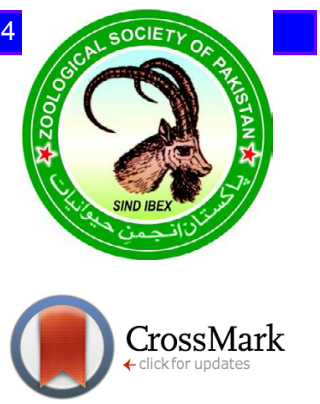

$\longrightarrow$

\section{INTRODUCTION}

$\mathrm{C}$ hinese mitten crab (Eriocheir sinensis), also known as crab, is an important economic aquatic source (Du et al., 2017). Molting is a critical physiological characteristic of crab. Precocity and molting abnormalities may cause huge economic losses. The study on molting mechanism of E.sinensis has been an attractive topic in aquatic crustaceans. Previous studies showed that molting is regulated by a variety of hormones, mainly coordinated through the interplay between molt-inhibiting hormone (MIH) secreted from the X-organ/sinus gland complex (XO$\mathrm{SG}$ ) in eyestalk and ecdysteroids secreted from Y-organ in cephalothorax (Techa and Chung, 2015). Sun et al. (2001) observed ultrastructure of sinus gland and confirmed the regulation foundation for gonadal development from the morphological structure. Wang et al. (2002) obtained eyestalks specific cDNA. Yao et al. (2006) constructed molt-inhibiting hormone prokaryotic expression vector, and prepared corresponding polyclonal antibodies. Sun et al. (2011) cloned full-length cDNA from molt-inhibiting hormone of eyestalks and discussed the expression of

\footnotetext{
Corresponding authors: nqjnqj1964@163.com; joyht2001@163.com; jinshsun@163.com 0030-9923/2018/0005-1777 \$ 9.00/0 Copyright 2018 Zoological Society of Pakistan
}

MIH mature peptide gene recombination in E. sinensis. On the study of ecdysone, Zhao and Lu (2004) observed ultrastructure of Y-organ during the molting cycle. He et al. (2013) constructed the expression vector of hormone regulated protein by the method of homologous recombination and purified ecdysone regulatory proteins subsequently. Gong et al. (2014) cloned full-length cDNA sequences of ecdysone receptor gene from Y-organ and analyzed the structure and tissue expression. These results laid a foundation for elucidating the molting mechanism of E. sinensis.

However, studies focusing on several or single genes/ proteins are not sufficient to completely understand the molting mechanism of crab. Protein-protein interactions are the basis of almost all biological processes. Therefore, It is necessary to identify possible interactions between proteins for the investigation of molting process (Mering et al., 2002). With the development of high-throughput detection technology such as yeast two-hybrid (Y2H) (Uetz and Hughes, 2000), and Tandem affinity purification (TAP/MS) (Gavin et al., 2002), which provide a vast amount of proteomic data. The protein-protein interaction networks (PINs) have been reconstructed for many species, including bacteria (Butland et al., 2005; Arifuzzaman et al., 2006), virus (Blasche et al., 2013), plants (GeislerLee et al., 2007; Rodgers-Melnick et al., 2013), animals (Guruharsha et al., 2011; Li et al., 2004) and humans 
(Ewing et al., 2007; Rolland et al., 2014), which providing a novel method for understanding the cellular mechanism in intracellular signaling, gene regulation and biochemical processes (Hao et al., 2016). It is a new development for the study of molting mechanism and investigation of the physiology processes in aquatic crustacean species with the PPI network from the systematic view.

Our group have reconstructed a PIN of E. sinensis which included 3,223 proteins and 35,787 interactions based on the transcriptomics sequencing of RNAs in eyestalk, Y-organ and hepatopancreas and the combination of protein-protein interaction information of six models organisms (Hao et al., 2014). In this work, the subcellular location of unidentified proteins was firstly annotated based on the previously reconstructed PIN of E. sinensis, and the molting interaction network was extracted from the global PIN. By analyzing the subcellular annotation of the molting interaction network, we provide a theoretical basis for the further elucidation of the molting mechanism in E. sinensis.

\section{METHODS}

Subcellular localization of unidentified proteins based on the neighbor nodes

Based on gene ontology (GO) database which contains the cellular components annotation information, the subcellular location of unidentified protein was annotated according to the location of neighbor proteins referring to the method of Alexei Vazquez's work (Vazquez et al., 2003). Firstly, for a certain protein, namely a "node" in the network, the number of its neighbor nodes with GO subcellular location annotation(s) (n) and the number of proteins $(\mathrm{m})$ that was annotated by each GO item was calculated. Once $\mathrm{m} / \mathrm{n}$ reached a critical value, the corresponding GO was annotated to this unidentified protein (Fig. 1) (Vazquez et al., 2003). In a network with an average degree more than 20, 20 neighbor proteins are unlikely to be annotated by the same GO, so it is necessary to set a proper threshold.

In this work, we set the threshold value as $75 \%$, that is, in all the GO annotations corresponding to the neighbor nodes of a unidentified protein, when a GO annotation satisfies $m / n \geq 75 \%$, this GO annotation will be added to the unidentified protein. When an unidentified protein has only one annotated neighbor node, all GO annotations of the neighbor node will be added to this protein. Detailed steps are as follows: (1) Obtain the protein-protein interaction information and identify the relationship between unidentified and identified proteins with GO cellular component annotations, namely subcellular locations, from the reconstructed PIN; (2) calculate the number of annotated neighbor proteins (m) of each unidentified protein and the number of proteins (n) annotated by each GO item; (3) Determine if the number of neighbor nodes with GO annotations is 1 , if the value equals to 1 , assign all functional annotations to the unidentified proteins; otherwise, calculate $\mathrm{m} / \mathrm{n}$ starting from any GO item, if $\mathrm{m} / \mathrm{n} \geq 75 \%$, then the GO annotation was given to the unidentified protein, otherwise go to the determination of next GO item; and (4) Repeat steps 1-3 until the number of GO-annotated proteins in the network does not change. It should be noted that after several iterations there may still exist the proteins that are not yet annotated.

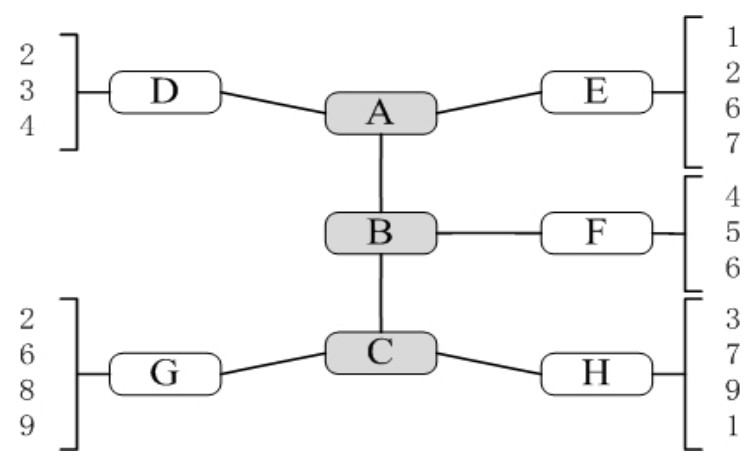

Fig. 1. Schematic diagram of unidentified protein annotation. The gray boxes represent unidentified proteins. The white boxes indicate proteins with known GO cellular component annotations. The numbers 1 to 9 indicate different GO items.

As shown in Figure 1, in the first loop, the number of neighbor proteins with GO annotations (D and E) interacting with unidentified protein $A$ is 2 , the GO annotation of protein $\mathrm{D}$ is 2, 3, 4 and the GO annotation of protein $\mathrm{E}$ is $1,2,6$, and 7, only the annotation 2 in all the functional annotations of the neighbor proteins satisfies the condition of $\mathrm{m} / \mathrm{n} \geq 75 \%$, then annotation 2 was annotated to protein A. Similarly only annotation 9 satisfies the condition of $\mathrm{m} / \mathrm{n} \geq 75 \%$ among all GO annotation of neighbor proteins interacting with unidentified protein $\mathrm{C}$, and hence the annotation 9 is assigned to the protein $\mathrm{C}$. For protein $\mathrm{B}$, only one neighbor node $\mathrm{F}$ has $\mathrm{GO}$ annotations, thus all of the GO annotations 4, 5, and 6 of node $F$ are assigned to protein B. In summary, After the first cycle of annotation, the annotations of protein $\mathrm{A}$ is annotation 2 , of protein $\mathrm{B}$ are annotation $4,5,6$, and of protein $\mathrm{C}$ is annotation 9. In the second cycle, protein $\mathrm{A}$ has three annotated neighbor proteins $\mathrm{B}, \mathrm{D}, \mathrm{E}$, according to the same principle, annotations 4 and 6 was added to protein A, that is, protein A has three annotations 2, 4 and 6 in total. Similarly, annotation 6 is added to protein $\mathrm{C}$, hence protein $\mathrm{C}$ has two annotations 6 and 9. The annotations were iteratively executed until no new annotation can be added to any unidentified protein. 


\section{Reconstruction of the molting interaction network}

In the previous study, we have reconstructed a PIN of E. sinensis (Hao et al., 2014) based on the transcriptomics sequencing of RNAs in eyestalk, Y-organ and hepatopancreas and the combination of proteinprotein interaction information of six models organisms obtained from PINA (Protein-Protein Interaction Network Analysis) database (Cowley et al., 2012). The network contains 3223 proteins and the biological process of 3045 proteins was annotated. Gene Ontology annotation has the characteristics of a hierarchical structure in which the molting process (GO: 0022404, molting cycle process), which is the highest-level keywords appear to molt, located in the fourth level of the entire GO hierarchy. So we took the GO biological process annotations of all the 3045 proteins to trace back along the GO hierarchy, and eventually the proteins which were able to go back to GO: 0022404 were considered as the molting proteins. Subsequently, the interactional relationships corresponding to the molting proteins were extracted from the PIN of $E$. sinensis, forming the molting interaction network. The network was visualized by Cytoscape software.

\section{Subcellular localization of molting-related proteins}

We searched for molting proteins in the global PIN of E. sinensis, and matched the subcellular location annotations from the global network to the corresponding molting proteins. The subcellular annotations were traced back along the GO hierarchical structure, and the upper subcellular location attribution was analysis.

\section{RESULTS}

\section{Subcellular location annotation of unidentified proteins}

According to transcriptomics sequencing results of Chinese mitten crab, the PIN contains 3223 proteins in which 2320 have subcellular localization annotations, accounting for $72 \%$ of the total proteins. These proteins correspond to a total of 4077 annotations. The rest of 903 proteins in the PIN are unidentified proteins which lack of subcellular location annotation. After three times iterative calculation for subcellular locations of unidentified proteins with the neighbor nodes based annotation method described in the method section, the subcelluar location of 830 proteins were obtained, accounting for $91.9 \%$ of the unidentified proteins. The locations for the rest of 73 proteins were unable to be annotated with this method. After annotation, the PIN of Chinese mitten crab contains 3150 proteins which have subcellular location annotations, accounting for $97.7 \%$ of the entire network with an increase of $27.7 \%$ compared to the PIN before annotation. The annotation results are shown in Figure 2.

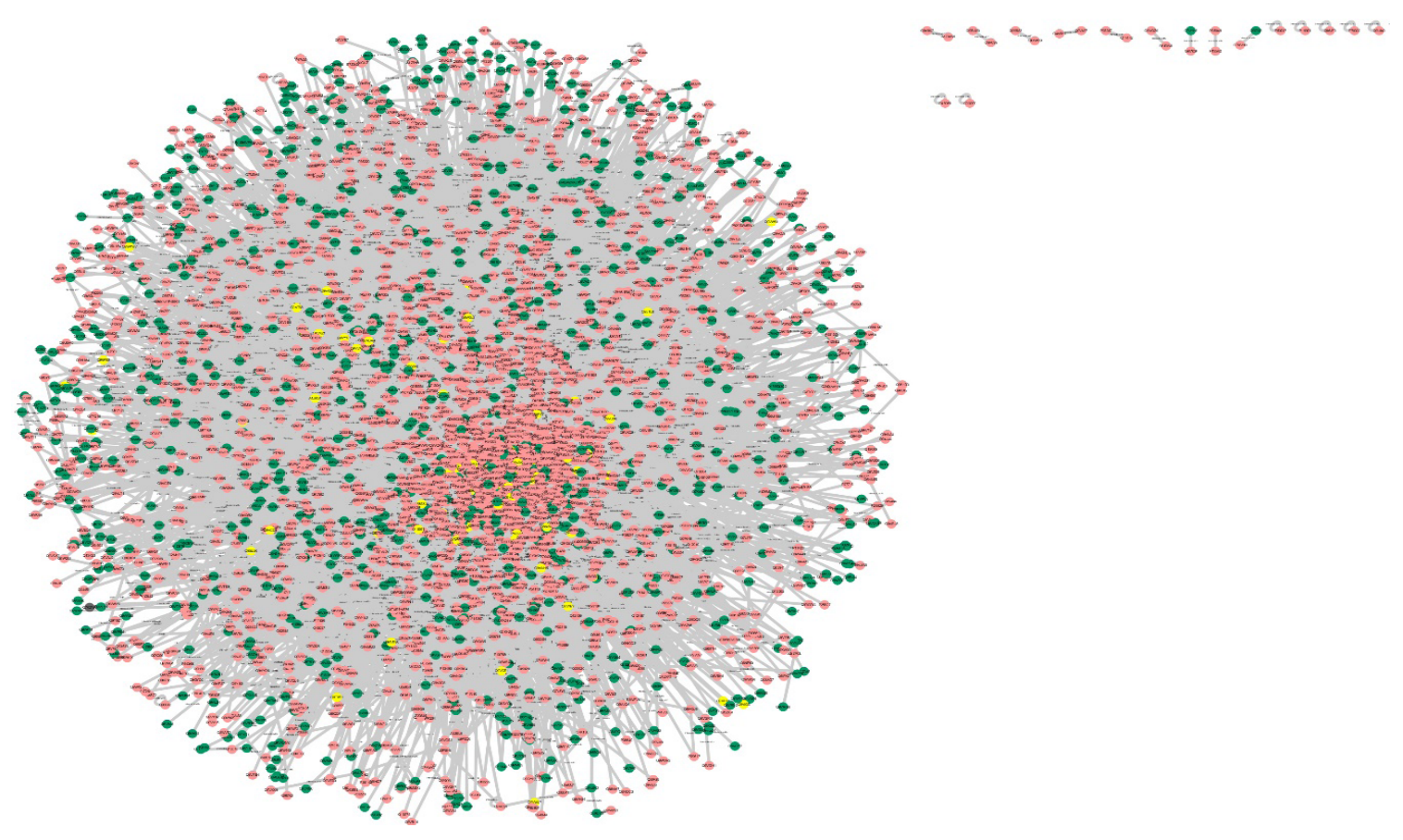

Fig. 2. Results of subcellular location annotation. Red indicates proteins with subcellular location annotations in the global network originally. Green indicates the proteins annotated in this work. Yellow indicates proteins that cannot be annotated with subcellular locations. 


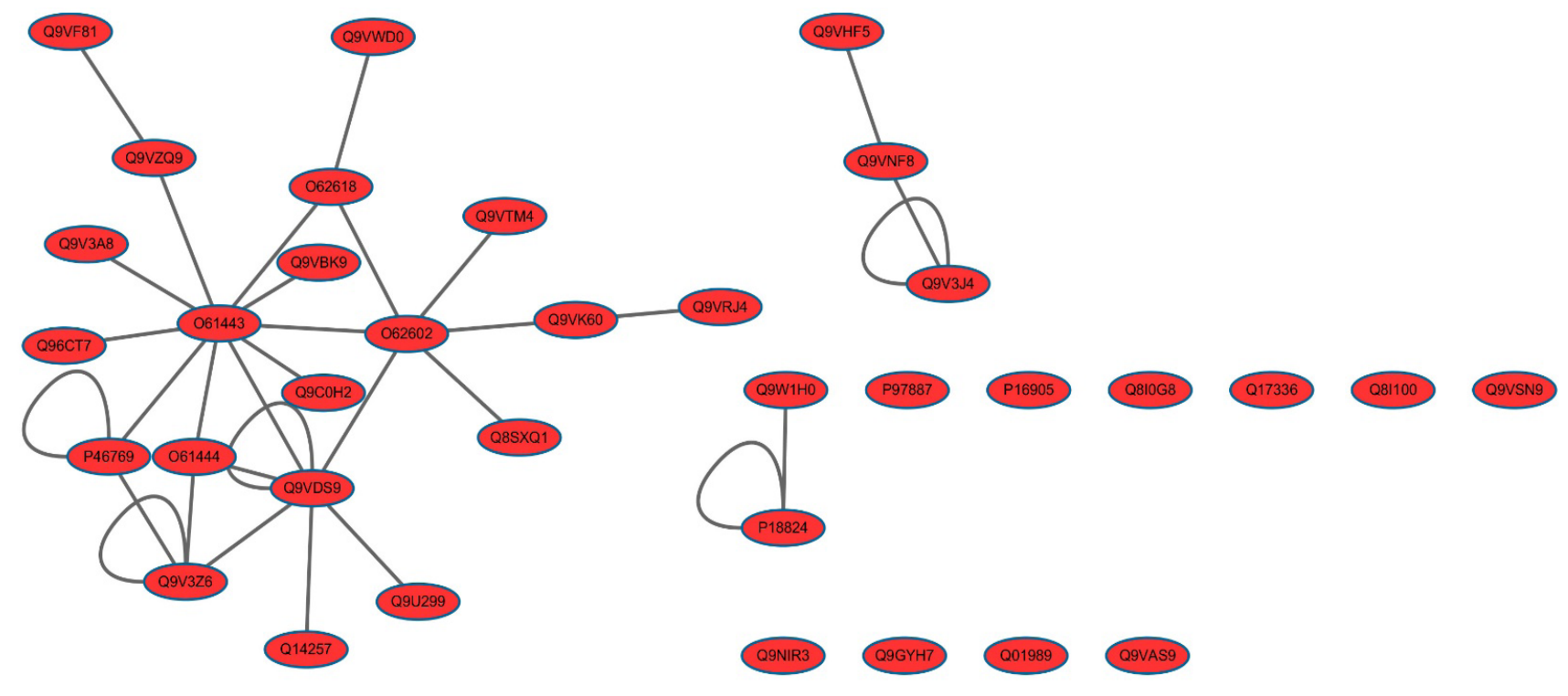

Fig. 3. PIN of molting protein of E. sinensis.

Table I.- Sub cellular location of molting protein annotation.

\begin{tabular}{ll}
\hline Protein & Subcellular location \\
\hline Q17336 & GO:0043231; GO:0030529 \\
P46769 & GO:0005737; GO:0030529 \\
Q9VWD0 & GO:0005924; GO:0031674 \\
Q01989 & GO:0005884; GO:0016023; GO:0016461; \\
& GO:0015630; GO:0045171 \\
Q9VAS9 & GO:0044464; GO:0043005; GO:0044424 \\
Q9V3Z6 & GO:0044448; GO:0042995; GO:0015629; \\
& GO:0043232 \\
Q9VZQ9 & GO:0043231 \\
O62602 & GO:0044424 \\
Q9U299 & GO:0031981 \\
Q9VSN9 & GO:0005768; GO:0044425 \\
Q9VNF8 & GO:0030133;GO:0044431; GO:0012507 \\
P97887 & GO:0030427; GO:0005764; GO:0031982; \\
& GO:0005783; GO:0043234; GO:0019866; \\
& GO:0044459; GO:0016021; GO:0005815; \\
& GO:0045202; GO:0030425; GO:0031965; \\
& GO:0000775; GO:0031674; GO:0005624; \\
& GO:0044297; GO:0044444 \\
\hline
\end{tabular}

Identification of molting protein and reconstruction of molting interaction network

Among all 3045 annotated protein which contains biological processes in the global network, by the method of GO backtracking, the GO annotation of these proteins were matched with the molting process (GO: 0022404). Finally 35 molt-related proteins which can be traced back to GO: 0022404 were obtained. These proteins include: O62618, Q9W1H0, O61444, O61443, Q8I0G8, P97887, Q9VDS9, O62602, Q9VNF8, Q9V3Z6, Q9v3J4, P46769, P16905, Q9VSN9, Q8I100, P18824, Q17336, Q01989, Q9VAS9, Q9NIR3, Q9GYH7, Q9U299, Q9VTM4, Q9C0H2, Q8SXQ1, Q9V3A8, Q96CT7, Q9VRJ4, Q9VZQ9, Q9VK60, Q9VF81, Q9VBK9, Q9VWDO, Q14257 and Q9VHF5. 32 interactional relationships were found corresponding to these molting proteins from the global PIN of E. sinensis. The molting network was comprised of the molting proteins and their interactions (Fig. 3), of which 10 proteins did not have any interactions involved. Actually, these proteins may interact to proteins with other functions in the global network.

\section{Cellular localization of molting proteins}

Totally 12 proteins involving 40 subcellular localization annotations were obtained by matching the 35 molting proteins to the global PIN and extracting corresponding subcellular location information (Table I). These GO annotations were backtracked through GO hierarchy and their upper level subcellular locations were analyzed. The proteins mainly distributed in the membrane, cytoskeleton, Golgi apparatus, vacuole, nucleus, cell-substrate junctions, neuron projection and ribonucleoprotein complex (Table II). The annotation of protein 062602 is intracellular part but the detailed location cannot be determined. 
Table II.- Distribution of molt protein subcellular location.

\begin{tabular}{ll}
\hline GO annotation & Protein \\
\hline Cell membrane & Q9VZQ9, Q9U299, Q9VSN9, \\
& Q9VNF8, P97887, Q9VWD0 \\
Cytoskeleton & Q9V3Z6 \\
Golgi apparatus & Q9VNF8 \\
Vacuole & P97887 \\
Nucleus & Q9U299, Q01989 \\
Sticky attachment connecting & Q9VWD0 \\
portion & \\
connecting portion & Q9VAS9 \\
Intracellular & O62602 \\
Ribosomal protein complexes & Q17336, P46769 \\
\hline
\end{tabular}

\section{DISCUSSION}

In all the localized molting proteins, half of the proteins (6 of 12) were located in membrane. It indicates that molting process is inextricably linked with the change of membrane, which is in consistence with Webster (1993) work that the binding site of MIH is the plasma membrane of Y-organ detected with the ligand blotting assay in Carcinus maenas.

In addition, new skin formation is an important stage in the process of molting, comprising of the growing and extending of subcutaneous cells, secreting epidermis and pigment layer which constitute the new skin.

The membrane plays an essential role in the cell growth, elongation and other morphological changes. Therefore, we speculate that a number of molting proteins locate on the cell membrane or affect cell-substrate junction to promote the new skin generation by regulating the cell membrane changes in the molting cycle. Subsequently the cytosol was excreted into the space between the old and new skins to crack the old skin cells and nucleus. This digestion process continues until all the membranes and most of the calcification layers were destroyed. As cell membrane is the protective barrier of cells, in order to destroy the cell structure and digests the cell, the first step of molting is to destroy the membrane structure, in which the molting proteins may play roles in the membrane to ensure the smooth running of molting process.

The hepatopancreas of crustaceans is an important organ for nutrient digestion and storage. It is closely related to the growth, development and reproduction of crustaceans. Digestion, absorption, storage and excretion of nutrients are achieved through the synthesis and secretion of digestive enzymes. The hepatopancreas cells of E. sinensis mainly consist of R, B, F and E cells (absorbing cells, vesicular cells, fibroblasts, and tubule ends blastocyte). These cells are periodically changed during molting (Tian et al., 2013). E cell is undifferentiated blastocyte which can differentiate to form R, B and F cells. E cell can also differentiate to other cells types through mitosis in which the cytoskeleton proteins are involved. The function of B cell is to transfer metabolic wastes into its large vacuoles after the absorption of nutrients. The molting proteins in vacuoles may participate in the transport of metabolic wastes.

Besides, the activity of digestive enzymes in hepatopancreas is also compatible with the molting cycle of E. sinensis. The crustaceans are fast and lurking motionlessly during the molting process. Because vast amount of energy will be consumed during the ephemeral molting period, a large number of energy substances (carbohydrates, lipids and proteins) will be accumulated in hepatopancreas in the proecdysis and anecdysis. Therefore, the quantity and activity of hepatopancreas amylase and trypsin increase significantly in order to digest and absorb the carbohydrates and protein substances (Kang et al., 2012). The synthesis and secretion of these two enzymes are relevant to the nuclear genetic transcription proteins, ribonucleoproteins and Golgi membrane proteins. So the proteins located in nucleus, ribonucleoprotein complex and Golgi apparatus can be found in the immune PIN.

The protein Q9VAS9 is located in the neuron projection, which may be related to the regulational complexity of molting in crustaceans. Molting process is regulated by both the nervous system and the endocrine system. Studies have shown that molting in crustaceans is regulated by a variety of hormones, mainly coordinated through molt-inhibiting hormone (MIH) secreted from the $\mathrm{X}$-organ/sinus gland complex (XO-SG) and ecdysteroids secreted from Y-organ (YO). XO-SG is the main control center of crustaceans neuroendocrine. SG, which consists of many axons of neurosecretory cell, is a neurological circulatory organ with the function of hormone storage and release. SG do not actually synthesize hormones itself (Zhu and Li, 2001). Most researchers believe that MIH regulates molting process by controlling YO to secret molting hormone (Lee et al., 2007), but the mechanism of MIH regulation is still unclear. Proteins in neuron projection may be involved in the hormone delivery process.

\section{CONCLUSIONS}

Molting process of the Chinese mitten crab is participated by a number of proteins, which interact with each other to form an interaction network. In this work, we 
extracted the molting PIN from the reconstructed $E$. sinensis PIN and further localized the molting proteins through the subcellular location annotation of proteins in E. sinensis PIN. Systematic localization and analysis of the molting PIN provide a theoretical basis for the elucidation of the molting mechanism, and further profit the development of Chinese mitten crab cultivation industry.

\section{ACKNOWLEDGMENTS}

This work was supported by the grants of the Major State Basic Research Development Program of China (program 973, 2012CB114405), the National Natural Science Foundation of China (31770904) and Tianjin Development Program for Innovation and Entrepreneurship.

\section{Statement of conflict of interest}

The authors declare that there is no conflict of interests regarding the publication of this article.

\section{REFERENCES}

Arifuzzaman, M., Maeda, M., Itoh, A., Nishikata, K., Takita, C., Saito, R., Ara, T., Nakahigashi, K., Huang, H.C., Hirai, A., Tsuzuki, K., Nakamura, S., Altaf-Ul-Amin, M., Oshima, T., Baba, T., Yamamoto, N., Kawamura, T., Ioka-Nakamichi, T., Kitagawa, M., Tomita, M., Kanaya, S., Wada, C. and Mori, H., 2006. Large-scale identification of protein-protein interaction of Escherichia coli K-12. Genome Res., 16: 686-691. https://doi. org/10.1101/gr.4527806

Blasche, S., Wuchty, S., Rajagopala, S.V. and Uetz, P., 2013. The protein interaction network of bacteriophage lambda with its host, Escherichia coli. J. Virol., 87: 12745-12755. https://doi. org/10.1128/JVI.02495-13

Butland, G., Peregrin-Alvarez, J.M., Li, J., Yang, W., Yang, X., Canadien, V., Starostine, A., Richards, D., Beattie, B., Krogan, N., Davey, M., Parkinson, J., Greenblatt, J. and Emili, A., 2005. Interaction network containing conserved and essential protein complexes in Escherichia coli. Nature, 433: 531537. https://doi.org/10.1038/nature03239

Cowley, M.J., Pinese, M., Kassahn, K.S., Waddell, N., Pearson, J.V., Grimmond, S.M., Biankin, A.V., Hautaniemi, S. and Wu, J.M., 2012. PINA v2.0: Mining interactome modules. Nucl. Acids Res., 40: D862-D865. https://doi.org/10.1093/nar/gkr967
Du, F., Li, Y., Wen, Z., Chen, R. and Xu, P., 2017. Development of simple sequence repeats (SSR) by Transcriptome in Chinese Mitten Crab (Eriocheir sinensis H. Milne Edwards). Pakistan J. Zool., 49: 497-503. https://doi.org/10.17582/journal. pjz/2017.49.2.497.503

Ewing, R.M., Chu, P., Elisma, F., Li, H., Taylor, P., Climie, S., McBroom-Cerajewski, L., Robinson, M.D., O'Connor, L., Li, M., Taylor, R., Dharsee, M., Ho, Y., Heilbut, A., Moore, L., Zhang, S., Ornatsky, O., Bukhman, Y.V., Ethier, M., Sheng, Y., Vasilescu, J., Abu-Farha, M., Lambert, J.P., Duewel, H.S., Stewart, I.I., Kuehl, B., Hogue, K., Colwill, K., Gladwish, K., Muskat, B., Kinach, R., Adams, S.L., Moran, M.F., Morin, G.B., Topaloglou, T. and Figeys, D., 2007. Large-scale mapping of human protein-protein interactions by mass spectrometry. Mol. Syst. Biol., 3: 89. https://doi.org/10.1038/ msb4100134

Gong, L., Zhang, Y.C., Sun, Y., Liu, Y.C., Geng, X.Y. and Sun, J.S., 2014. Cloning and expression analysis of ecdysteriod receptor gene (Ers-EcR) in Eriocheir sinensis (in Chinese). J. Fish China, 38: 786-793.

Gavin, A.C., Bösche, M., Krause, R., Grandi, P., Marzioch, M., Bauer, A., Schultz, J., Rick, J.M., Michon, A.M. and Cruciat, C.M., 2002. Functional organization of the yeast proteome by systematic analysis of protein complexes. Nature, 415: 141147. https://doi.org/10.1038/415141a

Geisler-Lee, J., O'Toole, N., Ammar, R., Provart, N.J., Millar, A.H. and Geisler, M., 2007. A predicted interactome for Arabidopsis. Pl. Physiol., 145: 317 329. https://doi.org/10.1104/pp.107.103465

Guruharsha, K.G., Rual, J.F., Zhai, B., Mintseris, J., Vaidya, P., Vaidya, N., Beekman, C., Wong, C., Rhee, D.Y., Cenaj, O., McKillip, E., Shah, S., Stapleton, M., Wan, K.H., Yu, C., Parsa, B., Carlson, J.W., Chen, X., Kapadia, B., VijayRaghavan, K., Gygi, S.P., Celniker, S.E., Obar, R.A. and Artavanis-Tsakonas, S., 2011. A protein complex network of Drosophila melanogaster. Cell, 147: 690-703. https://doi.org/10.1016/j.cell.2011.08.047

Hao, T., Zheng, Z., Wang, B., Zhang, Y.C., Liu, Y.C., Geng, X.Y. and Sun, J.S. 2014. The protein-protein interaction network of eyestalk, Y-organ and hepatopancreas in Chinese mitten crab Eriocheir sinensis. BMC Syst. Biol., 8: 417-422. https://doi. org/10.1186/1752-0509-8-39

Hao, T., Peng, W., Wang, Q., Wang, B. and Sun, J.S., 2016. Reconstruction and application of protein- 
protein interaction network. Int. J. mol. Sci., 17: 907. https://doi.org/10.3390/ijms17060907

He, C., Chen, P., Gao, X., Gao, L. and Li, L., 2013. Expression and purification of ecdysteroidregulated protein from Chinese mitten crab Eriocheir sinensis in E. coli. Mol. Biol. Rep., 40: 6987-6995. https://doi.org/10.1007/s11033-0132818-6

Kang, X.J., Tian, Z.H., Wu, J.L. and Mu, S.M., 2012. Molt stages and changes in digestive enzyme activity in hepatopancreas during molt cycle of Chinese mitten crab, Eriocheir sinensis (in Chinese). J. Fish. Sci. China, 19: 806-812.

Lee, S.G., Bader, B.D., Chang, E.S. and Mykles, D.L., 2007. Effects of elevated ecdysteroid on tissue expression of three guanylyl cyclases in the tropical land crab Gecarcinus lateralis: possible roles of neuropeptide signaling in the molting gland. J. exp. Biol., 210: 3245-3254. https://doi.org/10.1242/ jeb.007740

Li, S., Armstrong, C.M., Bertin, N., Ge, H., Milstein, S., Boxem, M., Vidalain, P.O., Han, J.D., Chesneau, A., Hao, T., Goldberg, D.S., Li, N., Martinez, M., Rual, J.F., Lamesch, P., Xu, L., Tewari, M., Wong, S.L., Zhang, L.V., Berriz, G.F., Jacotot, L., Vaglio, P., Reboul, J., Hirozane-Kishikawa, T., Li, Q., Gabel, H.W., Elewa, A., Baumgartner, B., Rose, D.J., Yu, H., Bosak, S., Sequerra, R., Fraser, A., Mango, S.E., Saxton, W.M., Strome, S., Van Den Heuvel, S., Piano, F., Vandenhaute, J., Sardet, C., Gerstein, M., Doucette-Stamm, L., Gunsalus, K.C., Harper, J.W., Cusick, M.E., Roth, F.P., Hill, D.E. and Vidal, M., 2004. A map of the interactome network of the metazoan $C$. elegans. Science, 303: 540-543. https://doi.org/10.1126/science.1091403

Mering, C.V., Krause, R., Snel, B., Cornell, M., Oliver, S.G, Fields, S. and Bork, P., 2002. Comparative assessment of large-scale data sets of proteinprotein interactions. Nature, 417: 399-403. https:// doi.org/10.1038/nature750

Rodgers-Melnick, E., Culp, M. and DiFazio, S.P., 2013. Predicting whole genome protein interaction networks from primary sequence data in model and non-model organisms using ENTS. BMC Genomics, 14: 608. https://doi.org/10.1186/14712164-14-608

Rolland, T., Tasan, M., Charloteaux, B., Pevzner, S. J., Zhong, Q., Sahni, N., Yi, S., Lemmens, I., Fontanillo, C., Mosca, R., Kamburov, A., Ghiassian, S. D., Yang, X., Ghamsari, L., Balcha,
D., Begg, B.E., Braun, P., Brehme, M., Broly, M.P., Carvunis, A.R., Convery-Zupan, D., Corominas, R., Coulombe-Huntington, J., Dann, E., Dreze, M., Dricot, A., Fan, C., Franzosa, E., Gebreab, F., Gutierrez, B.J., Hardy, M.F., Jin, M., Kang, S., Kiros, R., Lin, G.N., Luck, K., MacWilliams, A., Menche, J., Murray, R.R., Palagi, A., Poulin, M.M., Rambout, X., Rasla, J., Reichert, P., Romero, V., Ruyssinck, E., Sahalie, J.M., Scholz, A., Shah, A.A., Sharma, A., Shen, Y., Spirohn, K., Tam, S., Tejeda, A.O., Trigg, S.A., Twizere, J.C., Vega, K., Walsh, J., Cusick, M.E., Xia, Y., Barabasi, A.L., Iakoucheva, L.M., Aloy, P., De Las Rivas, J., Tavernier, J., Calderwood, M.A., Hill, D.E., Hao, T., Roth, F.P. and Vidal, M., 2014. A proteomescale map of the human interactome network. Cell, 159: 1212-1226. https://doi.org/10.1016/j. cell.2014.10.050

Sun, J.S., Liu, A.X., Du, Y.Z. and Gu, J.L., 2001. Microstructure and ultrastructure of the sinus gland in the eyestalk of Eriocheir sinensis (in Chinese). Acta Zool. Sin., 47: 27-31.

Sun, Y., Zhang, Y.C., Liu, Y.C., Wang, X.H., Yufan, W., Xuyun, G., Jinsheng, S. and Weijun, Y., 2011. Cloning and expression analysis of molt-inhibiting hormone gene (Es-Mih) in Eriocheir sinensis (in Chinese). Acta Hydrobiol. Sin., 35: 210-217. https://doi.org/10.3724/SP.J.1035.2011.00210

Tian, Z.H., Kang, X.J. and Jiao, C.Z., 2013. Changes in cell type composition in the hepatopancreas of Chinese mitten crab Eriocheir sinensis during the molting cycle (in Chinese). J. Fish. Sci. China, 20: 1175-1181. https://doi.org/10.3724/ SP.J.1118.2013.01175

Techa, S. and Chung, J.S., 2015. Ecdysteroids regulate the levels of molt-inhibiting hormone (MIH) expression in the blue crab, Callinectes sapidus. PLoS One, 10: e0117278. https://doi.org/10.1371/ journal.pone.0117278

Uetz, P. and Hughes, R.E., 2000. Systematic and largescale two-hybrid screens. Curr. Opin. Microbiol., 3: 303-308. https://doi.org/10.1016/S13695274(00)00094-1

Vazquez, A., Flammini, A., Maritan, A. and Vespignani, A., 2003. A global protein function prediction in protein-protein interaction networks. Nat. Biotechnol., 21: 697-700. https://doi.org/10.1038/ nbt825

Wang, Z.Z., Xiang, J.H. and Cui, Z.X., 2002. Molecular cloning and sequence analysis of cDNA encoding 
partial putative molt-inhibiting hormone from the crab Eriocheir sinensis (in Chinese). Oceanol. Limnol. Sin., 33: 432-438.

Webster, S.G., 1993. High-affinity binding of putative moult-inhibiting hormone (MIH) and crustacean hyperglycaemic hormone $(\mathrm{CHH})$ to membranebound receptors on the Y-organ of the shore crab Carcinus maenus. Proc. R. Soc. Lond. B: Biol., 251: 53-59.

Yao, Y., Zhou, K.Y. and Song, D.X., 2006. Expression and polyclonal antibody preparation of moltinhibiting hormone 1 (MIH1) from the mitten crab Eriocheir japonica sinensis (in Chinese). Acta Zool. Sin., 52: 209-214.

Zhao, W.X. and Lu, J.F., 2004. Ultrastructure of Y-organ in Eriocheir sinensis during molt cycle (in Chinese). J. Fish. Sci. China, 11: 74-77.

Zhu, X.M. and Li, S.J., 2001. Regulation of molting in crustacean larvae (in Chinese). J. Fish. China, 25: 379-384. 